\title{
City Magazines and Social Media: Moving beyond the Monthly
}

Susan Currie Sivek

Linfield College

Follow this and additional works at: https://digitalcommons.linfield.edu/mscmfac_pubs

Part of the Communication Technology and New Media Commons, and the Mass Communication Commons

\section{DigitalCommons@Linfield Citation}

Sivek, Susan Currie, "City Magazines and Social Media: Moving beyond the Monthly" (2013). Faculty Publications. Published Version. Submission 14.

https://digitalcommons.linfield.edu/mscmfac_pubs/14

This Published Version is protected by copyright and/or related rights. It is brought to you for free via open access, courtesy of DigitalCommons@Linfield, with permission from the rights-holder(s). Your use of this Published Version must comply with the Terms of Use for material posted in DigitalCommons@Linfield, or with other stated terms (such as a Creative Commons license) indicated in the record and/or on the work itself. For more information, or if you have questions about permitted uses, please contact digitalcommons@linfield.edu. 


\title{
City Magazines and Social Media: Moving Beyond the Monthly
}

\author{
Susan Currie Sivek, Linfield College \\ susan.sivek@gmail.com
}

\begin{abstract}
City magazines have long been established in many American locales, but digital media of all types are now offering opportunities and challenges to this genre of magazine. Digital media have also complicated the rapidly changing ecosystems of local information in which urban citizens reside. The resources and popularity of newspapers and broadcast news have shifted, and other forms of print, digital, and mobile media are assuming important roles in informing the public. With all these factors at work, this may be a pivotal moment for city magazines as they explore innovative technology, creative business strategies, and the new possibilities for coverage that these offer. As part of a larger, multi-method project, this study analyzes to what degree city magazines are using Twitter to move beyond a monthly publication schedule, to activate and engage audiences in new ways, and to address local topics and concerns. This study reveals that city magazines are informing the public and engaging their audiences through social media in limited but promising ways. It also strongly suggests that these magazines may indeed be using technology to move beyond their traditional monthly publication schedule and become a more vibrant part of local audiences' information environment.
\end{abstract}

Keywords: city magazines, social media, Twitter

\section{Introduction}

City residents in need of information about their local communities have always had a variety of news sources to use: newspapers, television, radio, local blogs and websites, and city magazines. In recent years, however, all these news media have expanded their efforts to include social media. Audiences can now find a constant flow of local news and information on Twitter and Facebook, and then - at least in theory — can engage in conversation with journalists and each other about local topics.

City magazines' place in this information environment is increasingly complex. City magazines typically have reported on stories well after the fact, offering a longer view and a more deeply reported perspective. They have also served as supporters of local business and tourism. However, with today's unfettered access to a 24/7 audience through the Web and social media, city magazines may now be able to develop new types of reporting and audience engagement beyond a

Susan Currie Sivek, Ph.D., is an assistant professor in the Department of Mass Communication at Linfield College in McMinnville, Oregon. She teaches courses in media studies and media writing, and conducts research on magazines and the effects of technology on journalism. Sivek is also the magazine industry correspondent for PBS MediaShift. 
monthly publication schedule. Finding ways to capitalize on the city magazine's unique strengths—in terms of both content and production processes - in this changing environment will be critical in order to maintain audience interest in a world moving away from print formats. City magazines are currently exploring the ways in which their product can complement other news sources and become a unique resource for local audiences.

This study - one part of an ongoing, multi-method analysis of contemporary American city magazines - examines the ways in which social media, specifically Twitter, are changing city magazines' relationship to their audiences. The study assesses the degree to which city magazines are using Twitter, engaging with readers through this medium, and addressing topics of local concern via Twitter. This analysis of the role of social media is critical to a deeper understanding of the unique functions of city magazines in their cities, as well as to determining whether the function of the city magazine is currently at a pivotal moment of fundamental change. More broadly, this analysis builds our understanding of how information is produced, distributed, and consumed by news media and their audiences in local areas.

\section{Research on City Magazines and Their Local Role}

There has not been extensive scholarly research on city magazines in recent years, and no studies have yet been conducted on these magazines' digital efforts. Four studies were published in Journalism Quarterly between 1979 and 1982, but they are now three decades old. Each of these studies reported on surveys of city and regional magazine publishers that examined their motives and goals as local information providers. A typical report (Hayes, 1981) concluded that these magazine publishers sought to publicize and "champion" their local areas for readers, who were thought to subscribe because of their pride in their cities. Hynds (1979) found that these magazines also aimed to provide information not always provided in newspapers, including lifestyle, food, and entertainment coverage. The publishers also wanted to offer a different perspective from that found in newspapers. Finally, Fletcher and VandenBergh (1982) cited one magazine editor who stated that "Metropolitan or city magazines provide the only medium which has the capability of establishing the 'identity and flavor' of a market...to capture the true 'picture' of the market it serves" (p. 314). That picture is offered in a more durable, visually appealing, and upscale medium than newspapers or local television news provide.

Greenberg (2000) offers a more critical and recent look at the content of city magazines and its connection to ownership. Greenberg argues that when city and regional magazines have been purchased by larger media conglomerates (such as Disney, Emmis Communications, and News Corporation), they have typically become homogenized and made to fit a formula that defines the conglomerates' other publications. This formula includes "toned down and reduced editorial content, increased pages of advertising and lifestyle reporting, new 'special sections' filled with consumer reports, and encyclopedic high-end listing sections at the back" (p. 251). Greenberg suggests that this mix appeals to advertisers and encourages the blurring of advertising/editorial boundaries, while it also subtly suggests that readers are intended to be consumers as opposed to more broadly concerned citizens, within their local area. 
Whatever their ownership or their publishers' goals, city magazines are still one of the primary ways in which local audiences can learn about their cities and the major issues and events within them. As such, these magazines are still fundamentally journalistic products, even though some might claim they are primarily intended as entertainment. Moreover, these magazines are often prominently displayed in local retail outlets and assert their authority as local experts with frequent "Best of" or "Local Awards" issues. Because of their local significance and claim to local authority, it is worth taking a closer look at what city magazines actually do in their local areas, both in their print editions and in their outreach to online audiences through social media and other digital tools.

\section{The Changing Role of City Magazines}

Because city magazines exist within the larger local information environment of their cities alongside newspapers and other news sources, it is important to understand these magazines' different informational functions, economic standing, and management considerations. While newspapers have been the traditional source for in-depth local journalism, their dominance has faded steadily since the introduction of TV news and has recently been threatened even more fundamentally by the availability of myriad sources of information on the Internet. Yet while audiences can obtain national and global news from multiple online sources, local news gathering is still largely conducted by city newspaper journalists.

Newspapers, faced with declines in circulation and advertising revenue, have looked for ways to showcase the value of the information they provide to their communities. Some newspapers have recognized that their unique strength is precisely this local information and have chosen to emphasize this coverage in their allocation of resources and physical design. For example, the Denver Post decided in 2011 to publish local news on the front page of the paper and in the first part of Section A, relegating national and world news to later pages of the paper (Phelps, 2012). Editor Greg Moore said, "That's the unique, unduplicated content that you can only really get from your local newspaper.... [W] just took the opportunity to use that news hole in a more aggressive way to promote and showcase our local coverage" (quoted in Phelps, 2012).

Even with the occasional strategic shift in focus like the Post's, newspapers have faced massive losses in revenue and have had to lay off staffers in dramatic numbers. The number of full-time newsroom staffers at newspapers has declined 28\% since 2000 (Project for Excellence in Journalism, 2012b). The loss of resources, both human and financial, at newspapers has meant that they are likely less able to tackle in-depth, long-term stories. While the decline of newspapers has been reported in many sources, a recent Federal Communications Commission (FCC) report (2011) summarizes the financial fallout and its consequences for the information needs of local communities. Recent analyses cited in this report show that from 2006 to 2009, the newspaper industry reduced by over a quarter its annual spending on reporting and editing. These cutbacks have resulted in dramatic losses of local newspaper coverage of major issues of public concern, such as local and state politics, crime, and health concerns (FCC, 2011).

Broadly speaking, magazines' staffing levels have declined in recent years as well, a trend that likely also affected city magazines. However, as of the end of 2011, it appears that employment in the 
industry has stabilized (Project for Excellence in Journalism, 2012b). Magazines are generally better positioned to deal with economic crises and technological change. Because their employees are typically not unionized (for better or worse, from the employees' perspective), magazines can shed staff and rehire as needed, which also allows them to bring in staff with greater technological expertise and thereby adapt to new online opportunities. In addition, magazines' frequent use of freelance work permits more flexibility in assigning and paying for in-depth, long-term projects that can be published on a more malleable schedule, whereas newspapers' need to fill a daily news hole compels them to allocate their remaining staff to that ongoing task. Magazines' capability to address staffing issues flexibly is one characteristic that has helped them attract ongoing investment from publishers, particularly in the area of digital publication for tablets and other mobile media. Finally, magazines' existing design staff may be better prepared for the challenge of creating appealing visual products for digital media, such as tablet devices, than are newspapers' designers, although magazines' physical design may also just inherently be a more intuitive fit for these digital forms. All in all, the magazine industry has endured the recent economic turmoil more successfully than the newspaper industry and is better positioned in a variety of ways to continue to adapt well.

Technology changes, including online news and mobile media, have also affected audiences' support for print newspapers and suggest that city magazines may be poised to become an increasingly popular alternative source for local information if they work to accommodate audiences' changing media preferences. A recent Project for Excellence in Journalism (PEJ) study (2012a) found that $72 \%$ of survey respondents said they followed local news "closely" and that these individuals generally prefer the local newspaper as their information source. However, these readers are not the readers of the future. The same PEJ survey found that younger local news enthusiasts use a much wider variety of local news sources-more than four different sources per week, which could include traditional media, websites, and/or social media. Younger local news users are more open to obtaining their local information from other sources and would likely be a logical future audience for the expansion of city magazines and their online products.

But city magazines are not exempt from competition with mobile and other online media. For example, the availability of free, crowd-sourced restaurant reviews on websites and mobile applications like Yelp have made city magazines' restaurant reviews less distinctive, although the magazines would likely retort that the editorial judgment and voice of a trusted publication should be of greater value to audiences seeking food information. Entertainment news and other local event information can also be found for free through websites and apps, but again, readers may find city magazines' distinctive presentation of this information worth its cost. In general, the presence of these online sources may encourage city magazines to further distinguish their food and entertainment coverage from the freely accessible online information and to better communicate to audiences what the magazines perceive to be the greater value of their work.

As a whole, city magazines stand at a crossroads in today's local media scene. In many cases, they can hire staff or freelancers to do longer in-depth stories that many newspapers can no longer do. As such, they may choose to move beyond the narrow range of lifestyle topics to which their coverage has traditionally been restricted (as earlier research demonstrated). They may also be motivated to do so by the fact that some of their turf - the restaurant and entertainment information for which they 
have been known - has now been encroached upon by online sources. Moreover, city magazines are no longer limited to the print format and can reach audiences at all times, instantaneously, through their websites and various social media- tools for which they may already be better prepared, in terms of staffing, than newspapers are. Clearly, this is an intriguing set of circumstances for city magazines. Much change is possible, but the degree to which these publications will choose to shift their mission and format is as of yet unknown.

\section{The Potential of Social Media for City Magazines}

At the center of the set of circumstances described above is the key role of technology. In particular, social media have already expanded the ways that city magazines can reach out to their audiences and beyond. Indeed, social media have become an important way that all types of news organizations and journalists can reach their audiences. Other recent research (e.g., the review provided by Hermida, 2012) has traced the ways in which traditional local media have adopted social media, including Twitter, for purposes including distributing links to online news stories, information gathering, and conversing with the audience. Holcomb, Gross, and Mitchell (2011) found that $93 \%$ of traditional news organizations' tweets contained links that directed readers to their own news websites. The authors also observed that reporters were generally not using Twitter to gather information from audiences. However, the possibilities for news organizations' use of Twitter are much broader. Social media also enable audiences to participate more fully in the construction of the news, whether through casual suggestions, substantial citizen journalism, or occasional items of user-generated content (Hermida, 2012).

Among the variety of social media available to journalists and audiences, Twitter has been uniquely positioned for breaking news, offering (usually) publicly available tweets, photos, videos, and links from around the world during major news events. Its brief 140-character message format also encourages easy conversation, and its low-stakes "follower/following" relationships enable users to build wide-ranging, diverse personal networks without the emotional implications of Facebook's "friending."

Twitter also presents special opportunities for city magazines that differ from newspapers' uses of social media. City magazines' more personal tone lends itself well to casual communications in tweets, whereas the obligations of journalistic distance compel most newspapers' organizational Twitter feeds to maintain a straightforward tone. City magazines also have the staffing flexibility, as described above, to allocate editorial staff to managing social media on behalf of their publications. This assignment may be more difficult at a daily newspaper, where tweeting is likely to be perceived by overworked reporters as an additional burden in an already strained work environment.

Finally, city magazines can activate their audiences on Twitter through the easy retweeting (resharing) of their posts in order to better publicize local events. Although Twitter is, of course, a global network, many users likely have geographically concentrated follower connections because of pre-existing personal relationships. In fact, a recent analysis of Twitter relationships showed that "distance considerably constrains ties," with 39\% of Twitter connections occurring within a region "the size of a metropolitan area" (Takhteyev, Gruzd, \& Wellman, 2011, p. 81). This geographic 
concentration is impressive, considering that many Twitter users also follow national celebrities, national news sources, and other often physically distant users. The dissemination of city magazines' tweets among concentrated local audiences of Twitter users could be an especially valuable tool for city magazines seeking additional readers, website traffic, customers for partners' promotions, and contributors for crowd sourcing and contests.

As a whole, the immediate, constant, and surprisingly local nature of Twitter-combined with the shifts in local information sources represented by the decline of traditional news mediaoffers a variety of intriguing opportunities for technological adaptation at city magazines. Not only may city magazines now explore the possibilities of filling information gaps created by shrinking newspapers, but they can also use social media to break out of their monthly publication schedules to maintain constant contact and conversation with audiences.

Because city magazines' use of Twitter may therefore be a key to understanding whether and how they are adapting to this new local information environment and technology, this study examines their use of this medium to disseminate content and engage with audiences (through conversations and retweeting). The following research questions are addressed:

- RQ1: How are city magazines using Twitter to engage their local communities?

- RQ1a. What topics are city magazines tweeting about?

- RQ1b. How often do city magazines reply to or mention other Twitter users? Who are these other users?

- RQ1c. How often are city magazines mentioned by other Twitter users?

- RQ1d. How do city magazines use hashtags to create or join conversations?

- RQ2: How do city magazines' Twitter popularity and usage vary, if at all, based on other characteristics of the print magazines' readership and/or on the magazines' Twitter activity?

\section{Method}

This study used DiscoverText (2012), an online data-gathering service, to collect and sample Twitter feeds from 20 city magazines across the U.S., which were then coded and analyzed. As this study is part of a larger research project focused on various aspects of American city magazines, the same selection of magazines was repeated for this component of the project. That selection was created using the procedure below.

An initial list of city magazines that won awards from the City and Regional Magazine Association (CRMA) from 2007 to 2011 was composed through a review of the records of award winners on the CRMA website. Using the CRMA award-winning magazines as the population for this study was intended to ensure that the magazines ostensibly doing the "best" work, in terms of satisfying the journalistic norms valued by their peers, would be those studied in this analysis. Winners of only the following award types, all focused on editorial content and community engagement, were included in the initial list: General Excellence, Reporting, Personality Profile, Feature Story, Reader Service, Civic Journalism, Community Service Project, Excellence Online, and 
Multimedia. All categories of the CRMA awards, which are given across three circulation ranges, were included in order to represent publications in both smaller and larger cities.

The list was then narrowed to include only city magazines, as opposed to state or regional magazines. Conveniently, this narrowed list created a set of 20 magazines for further analysis. The final list of magazines also represents diversity in other key factors, including city size, geographic region, circulation, and ownership (independent vs. chain). The diversity of magazines included in this study is important, as magazines' content, technological savvy, and resources may differ based on city size, ownership, and popularity. Table 1 provides a list of the magazines included in the study, their cities' population, their print circulation, the Twitter account used for analysis in this study, and the number of Twitter followers and average tweets per day for each as of September 2012.

Table 1

Magazine Circulation and Twitter Data

\begin{tabular}{|c|c|c|c|c|c|}
\hline $\begin{array}{l}\text { Magazine } \\
\text { Name }\end{array}$ & $\begin{array}{c}\text { Primary Twitter } \\
\text { Account }\end{array}$ & Circulation* & $\begin{array}{c}\text { US Census } \\
\text { Local } \\
\text { Population }\end{array}$ & $\begin{array}{l}\text { Twitter } \\
\text { Followers }\end{array}$ & $\begin{array}{c}\text { Tweets/Day } \\
\text { Average }^{* *}\end{array}$ \\
\hline 5280 & @5280magazine & 77,027 & 610,345 & 18,082 & 3.8 \\
\hline $\begin{array}{l}\text { Atlanta } \\
\text { Magazine }\end{array}$ & @AtlantaMagazine & 66,996 & 540,922 & 17,705 & 2.4 \\
\hline Baltimore & @baltimoremag & 53,145 & 637,418 & 12,430 & 2.9 \\
\hline Boston & @bostonmagazine & 110,390 & 645,169 & 27,884 & 2.1 \\
\hline Charlotte & @CharlotteMag & 35,000 & 704,422 & 9,198 & 1.2 \\
\hline Chicago & @chicagomag & 129,199 & $2,851,268$ & 35,956 & 5.9 \\
\hline Cincinnati & @cincinnatimag & 37,426 & 333,012 & 5,527 & 0.6 \\
\hline D Magazine & @dmagazine & 22,000 & $1,299,542$ & 24,004 & 4.4 \\
\hline $\begin{array}{l}\text { Evansville } \\
\text { Living }\end{array}$ & @Evansville & 13,000 & 116,584 & 1,171 & 0.6 \\
\hline Honolulu & @HonoluluMag & 35,000 & 390,738 & 12,024 & 4.1 \\
\hline Hour Detroit & @Hour_Detroit & 45,000 & 910,921 & 12,783 & 0.6 \\
\hline $\begin{array}{l}\text { Indianapolis } \\
\text { Monthly }\end{array}$ & @indymonthly & 41,000 & 820,445 & 7,652 & 1.2 \\
\hline Los Angeles & @LA_mag & 140,000 & $3,831,868$ & 27,634 & 4.4 \\
\hline Madison & @madison_mag & 20,833 & 235,419 & 4,628 & 1.2 \\
\hline Memphis & @memphismagazine & 22,500 & 676,640 & 1,429 & 0.6 \\
\hline MPLS St. Paul & @mspmag & 17,710 & $2,968,806$ & 17,714 & 2.3 \\
\hline Philadelphia & @phillymag & 116,840 & $1,547,297$ & 19,403 & 3 \\
\hline $\begin{array}{l}\text { Portland } \\
\text { Monthly }\end{array}$ & @pomomagazine & 52,892 & 566,143 & 9,780 & 2.3 \\
\hline
\end{tabular}




\begin{tabular}{llrrrr} 
Seattle Met & $@ S e a t t l e M e t$ & 55,430 & 616,627 & 14,634 & 7.7 \\
Washingtonian & @washingtonian & 137,002 & 599,657 & 35,808 & 6.2 \\
\hline
\end{tabular}

* Based on most recent media kits available through magazines' websites.

** Calculated using howoftendoyoutweet.com service.

DiscoverText was configured to retrieve all tweets sent from these magazines' official, primary Twitter accounts, as well as all other tweets from any Twitter user in which the magazines' Twitter usernames were used in any context. Collection occurred for about eight consecutive weeks in the summer of 2012. The final archive contains 31,639 tweets. Of those, 6,640 tweets were sent from the magazines' own accounts; the other 24,999 were tweets that mentioned the magazines' Twitter usernames.

Metadata about the entire tweet archive was available through DiscoverText's utilities, including hashtag frequency, username mention frequency, and other key information about the tweets. Specific uses of these metadata will be described in more detail below.

In addition to these metadata, a stratified random sample was constructed from the entire archive that consisted of $20 \%$ of the tweets sent from each magazine's account, resulting in 1,334 tweets. This sample was further analyzed by two coders (the researcher and a trained undergraduate research assistant). Each tweet was manually coded for its specific topic, using a custom codebook built within the Web-based DiscoverText coding system. Intercoder reliability (Cohen's kappa) averaged .88 for the topic variable.

\section{Results}

This analysis of city magazines' Twitter efforts reveals that the magazines are using this medium primarily to distribute information about local entertainment and food options, as might be expected based on their print editions' typical content. The magazines are also perhaps underutilizing the unique strengths of Twitter-its multimedia, conversational, and linking capabilities — as well as its capacity for promotion. The magazines' social media efforts are strongly tied to daily and weekly events, however, suggesting that they are moving beyond their usual monthly schedule to reach audiences much more frequently and to direct them primarily to their own online content. The magazines are also, to a limited degree, beginning to engage more geographically widespread conversations through their use of hashtags. Finally, the magazines tend to draw more Twitter followers when they possess large print circulations and tweet often.

\section{Research Question 1: City Magazines' Use of Twitter}

Topics. The human-coded sample of 1,328 tweets from city magazines contained tweets on a wide range of topics - from restaurants to weddings, sports to local history. However, tweets about arts and entertainment topics (e.g., local concerts) and restaurants, food, and drink dominated these magazines' Twitter feeds, constituting $23.5 \%$ and $21.1 \%$ of their tweets respectively. All other topics constituted roughly the remaining half of the sampled tweets, as shown in Table 2. Public affairs topics 
were represented infrequently, with politics-related tweets constituting only $5.4 \%$ of the sample.

Although some of the topics listed in the table were represented by only a small number of tweets, they are still included separately to note their relative rarity. It is surprising to see, for example, how rarely the city magazines linked to online photos or videos of interest to their local audiences and how rarely they addressed local shopping and business. Videos and photos are extremely popular with social media users, and local shopping and business are covered rather frequently in city magazines' print editions (as a complementary portion of this research project has shown). Local shopping and business topics might also offer opportunities for e-commerce partnerships and promotions, but the city magazines do not appear to be pursuing these often.

\section{Table 2}

Topics of Sampled City Magazine Tweets $(n=1,328)$

\begin{tabular}{lcc}
\hline Topic & Frequency & Percentage of Sample \\
\hline Entertainment and arts & 312 & $23.5 \%$ \\
Food, drink, and restaurants & 281 & $21.1 \%$ \\
Reply to Twitter user ([.]@username begins tweet) & 105 & $7.9 \%$ \\
Other topics & 79 & $6.0 \%$ \\
Sports & 78 & $5.9 \%$ \\
Politics & 71 & $5.4 \%$ \\
Travel (i.e., local/regional destinations) & 51 & $3.8 \%$ \\
Business & 42 & $3.2 \%$ \\
Magazine print edition announcement & 41 & $3.1 \%$ \\
Fashion & 38 & $2.9 \%$ \\
Local awards (e.g., "Best of...”) & 36 & $2.7 \%$ \\
Crime and mystery & 30 & $2.3 \%$ \\
Magazine contest announcement & 28 & $2.1 \%$ \\
Magazine promotion (external company offer) & 26 & $2.0 \%$ \\
Local culture and history & 22 & $1.7 \%$ \\
Shopping & 20 & $1.5 \%$ \\
Transportation & 13 & $1.0 \%$ \\
Weddings & 12 & $0.9 \%$ \\
Home and garden & 11 & $0.8 \%$ \\
Education & 10 & $0.8 \%$ \\
Online photography & 70 & $0.8 \%$ \\
Environment and ecology & 5 & $0.6 \%$ \\
Online videos & $\mathbf{3} \%$ & $0.4 \%$ \\
Total & $\mathbf{1 0 0 . 4 \% *}$ \\
\hline$*$ The & \\
\hline
\end{tabular}

* The total is greater than $100 \%$ because of rounding. 
Moreover, the magazines are not using Twitter extensively to promote their own print products, facilitate reader contests, or engage in promotions with business partners. They are not even tweeting all that frequently about their local award competitions_- a prominent annual feature and event for most, if not all, of these publications. As many city magazines now seek readers' votes online for these competitions, it is surprising that they are not publicizing that effort via Twitter more frequently.

Finally, it is also worth noting the frequency of terms connoting timely topics in this archive of tweets. Within the complete archive of tweets from or mentioning these city magazines, there were numerous tweets using the terms "today" (1,727 occurrences), "tonight" (752), "this weekend" (960), "tomorrow" (413), "right now" (234), and "happy hour" (229). While city magazines have traditionally been published on a monthly or bimonthly schedule, clearly their Twitter efforts have taken on a much more immediate nature, reaching audiences constantly-not only between issues but even throughout the day — to guide their daily activities.

Interactions with other users. One goal of this project was to examine the quantity and type of interactions that city magazines carried out with other Twitter users. In the complete archive of the 6,640 tweets sent from the magazines' accounts, 2,213 mentioned a Twitter username (i.e., referred to it using Twitter @username format—such as @washingtonian—which informs the account holder of the mentioned username that he/she has been discussed and links to his/her account).

The top 10 usernames mentioned by each magazine were collated using the metadata provided by DiscoverText. These "mentions" (as they are called on Twitter) might include direct interactions with another user by the magazine or mentions of other users by username in a tweet from the magazine. These conversation partners' Twitter account profiles were examined and sorted into categories that described them (such as "local restaurant" or "national media organization").

This analysis revealed that the magazines' mentions of Twitter users were highly selfreferential. In fact, $71.8 \%$ of these "conversational" tweets contained either the magazine's own Twitter username as part of the text of the tweet, or they mentioned a magazine staffer's Twitter account or an affiliate product of the magazine (e.g., one of its own blogs). This means that a fairly low proportion, just over a quarter of the magazines' "conversational" tweets, actually mentioned a Twitter user not affiliated with the magazine. However, some of these tweets were actually direct replies to other Twitter users - truly conversational in nature. The DiscoverText metadata could not provide the frequency of such tweets in the entire archive. However, the sample of tweets that was human-coded did contain tweets that were clearly replies to other Twitter users sent from the magazines' accounts, and these were coded as such. In that sample, only about $8 \%$ of the magazines' tweets were replies to other users, as shown in Table 1 . In other words, actual "conversation" was rather infrequent.

Other users' discussion of the magazines. While city magazines' own tweets at times contain references to other Twitter users, as described above, the magazines themselves were also mentioned by other Twitter users. In fact, such discussion occurred in 24,999 tweets gathered in this archive. Other users' mentions of the magazines' Twitter usernames were collected and analyzed using the 
metadata provided by DiscoverText. The analysis revealed that almost half these mentions_-11,908 tweets-were more than likely simply retweets of the magazines' original tweets or resharing of the magazines' tweets using "via," another common Twitter attribution term. The tweets that used one of these terms in conjunction with a magazine's username were located by searching the entire tweet archive for these exact phrases (e.g., "RT @BostonMagazine,” “via @5280magazine”).

The other half of the mentions of city magazines in the remaining 13,091 tweets likely contains a variety of discussions of the magazines. However, these tweets could not be isolated for automated analysis using the selected data-collection service, and so additional human coding would be required to better understand the nature of Twitter users' discussions regarding these magazines. The extent of analysis possible for this large number of tweets in the current study was the creation of a word cloud to reveal which words were used most frequently in other users' mentions of the city magazines, excluding the retweets described above. Using the online tool wordle.net, a word cloud (see Figure 1) was created with the text of all the tweets that mentioned the magazines but that did not include "RT" or "via." The magazines' own account names and variations on them were removed from the word cloud. Perusing the final word cloud reveals a highly positive mood in tweets mentioning the magazines, with the words "best," "thanks," "great," "love," and "new" occurring frequently. Again, detailed human analysis of the tweets mentioning the magazines might reveal more about the nature of conversation about their work. However, this initial glimpse suggests that Twitter users are not only actively engaged in discussing these magazines online, but they also appear to feel positive toward them.

\section{Figure 1}

\section{Word Cloud of Terms Used in Twitter Mentions of City Magazines}

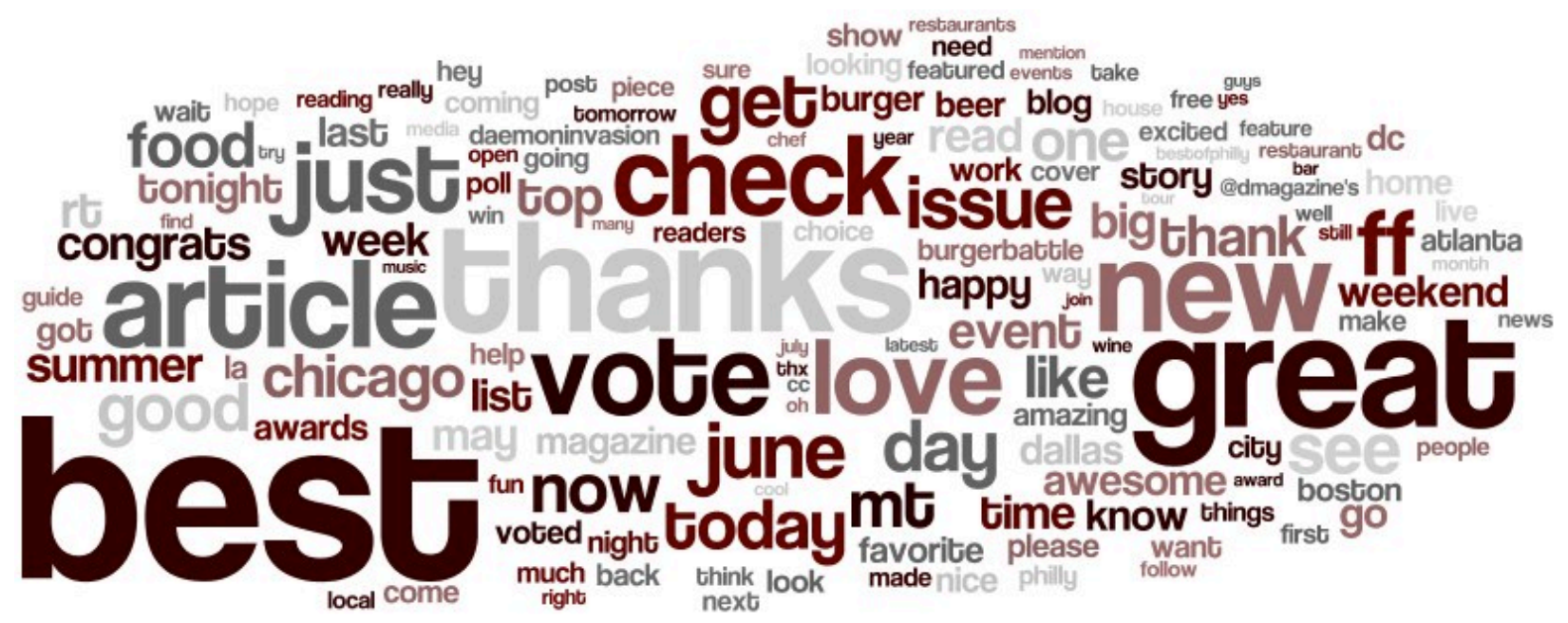

Magazines' use of hashtags. The hashtags used by the magazines in their tweets were gathered and ranked by frequency using the metadata provided by DiscoverText. The complete listing is shown in Table 3. 
Hashtags are terms marked with a \# to indicate keywords or topics in a tweet (Twitter, 2012). Users can click on a hashtag in any tweet to see a list of all current tweets that contain that hashtag from all Twitter users. In this way, hashtags facilitate conversations and even organized "chats" about specific topics without regard to existing follower/following relationships. In other words, they make widespread conversation on a topic possible across Twitter. Hashtags can be difficult to analyze when detached from their time of use and immediate conversational setting. Hashtags are often related to events in the news, as shown in Philadelphia's use of the "Sandusky" hashtag, related to the Jerry Sandusky case at Penn State. In addition, hashtags are extremely contextual. They may be used for humor or emphasis within a tweet, so they might not make sense when considered in isolation. However, it is still interesting to observe general trends in the hashtags used by these city magazines.

First, some city magazines create and regularly use their own hashtags to generate ongoing reference lists, so to speak, for their audience. For example, Twitter users who click on 5280's " 5280 BestBet" hashtag will retrieve a list of all tweets that list the magazine's (and other users') suggestions for entertainment and food. This recurring hashtag not only creates a dynamic reference tool for users, but it also becomes something of a recognizable brand name in itself.

\section{Table 3}

Top Four Hashtags Used More Than Once in All Tweets from City Magazines $(n=6,640)$

\begin{tabular}{ll}
\hline Magazine & Hashtags in Rank Order of Use \\
\hline 5280 & 5280BestBet, Denver, Colorado, Rockies \\
Atlanta & SummerGuide, atldining, atlarts, atlmodernism \\
Baltimore & events, restaurants, chefs, pier6 \\
Boston & CapeCod, Boston, MBTA, mapoli \\
Charlotte & clt, DNC2012, BOBawards, charlotte \\
Chicago & NATO, ChiSingles12, AfternoonShift, CRMA \\
Cincinnati & Reds, CRMA, Cincinnati, longreads \\
D Magazine (Dallas) & thingstodoindallas, BestofBigD, DallasTNT, thingstodo \\
Indianapolis Monthly & Indy500, IMDish, Indy, ACBW \\
Los Angeles & 101CheapEats, 52GreatWeekends, AfternoonCram, longreads \\
Madison & BoM2012 \\
Memphis & Memphis, constantcontact \\
MPLS St. Paul & HappyBdayMSP, MSPShowcaseHomes, steelechallenge, \\
Philadelphia & MNRestaurantWeek \\
Portland Monthly & LGBT, Sandusky, BestofPhilly, BeWellBootCamp \\
Seattle Met & Sasquatch, closerpdx, pdxcandide, pomolaf \\
Washingtonian & FF \\
\hline
\end{tabular}

Evansville Living, Honolulu, and Hour Detroit either used no hashtags during the archived time period or used none more than once. 
Second, the magazines are creating local conversations around hashtags they have created. For example, Chicago Magazine's \#ChiSingles 12 hashtag, created for its Chicago Singles 2012 competition and feature, generated significant buzz among its Twitter audience and was used frequently in the magazine's own tweets.

Third, many of these magazines are tying their tweets to larger discussions of their cities through local hashtags. The city names shown in the hashtag list above can be used by Twitter users anywhere who want to comment on and be part of a larger conversation about that city. Using \#Boston or \#Memphis in a tweet links that tweet to larger discussions about the city on Twitter.

Finally, the magazines are also reaching out to larger national and even global audiences through these hashtag selections. Hashtags like \#DNC2012 (for the 2012 Democratic National Convention in Charlotte, North Carolina) and \#Indy500 (for the Indianapolis 500 race) interject the magazines' tweets into the much bigger Twittersphere of conversations on those topics. Tweets with sports teams' names used as hashtags have the same effect. From a self-promotional angle, hashtags like \#longreads (a popular hashtag used to share quality long-form reading) and \#CRMA (for the City and Regional Magazine Association awards) suggest that the city magazines are seeking wider recognition of their quality work and awards.

\section{Research Question 2: Print Readership and Magazines' Twitter Activity}

In order to explore the relationships among other key characteristics of the magazines' readership and their Twitter activity, basic data about the magazines were compiled from their media kits, made available through their websites. In addition, the numbers of Twitter followers as of the time of writing were collected, as well as the average number of tweets sent per day from their accounts. (These data were provided in Table 1.) A variety of correlations were calculated using these data, and they are reported in Table 4.

As the table reflects, the size of a city magazine's local population has a moderate correlation to its number of Twitter followers. However, the number of subscribers to the print magazine correlates more strongly to its number of followers. 
Table 4

Summary of Pearson Correlations among Print Magazine Readership Characteristics

and Twitter Activity Measures $(n=20)$

\begin{tabular}{lcccccc}
\hline \multicolumn{1}{c}{ Magazine Characteristic } & $\mathbf{1}$ & $\mathbf{2}$ & $\mathbf{3}$ & $\mathbf{4}$ & $\mathbf{5}$ & $\mathbf{6}$ \\
\hline 1. Circulation & -- & $.451^{*}$ & $.818^{* *}$ & $.543^{*}$ & -.142 & .360 \\
2. Local Population & & -- & $.550^{*}$ & .312 & .004 & .325 \\
3. Number of Twitter & & & -- & $.697^{* *}$ & -.311 & $.576^{* *}$ \\
Followers & & & & - & & \\
4. Tweets/Day Average & & & & -.173 & .262 \\
5. Average Reader Age & & & & & -- & -.440 \\
6. Household Income & & & & & & -- \\
\hline
\end{tabular}

${ }^{*} p<.05$ (2-tailed)

${ }^{* *} p<.01$ (2-tailed)

In addition, the strongest correlation for the number of daily tweets sent by a magazine was with its number of followers, followed by its print circulation. This relationship implies that regardless of city size, a magazine's commitment to shaping and participating in a vibrant community of Twitter users may be most important in sustaining frequent engagement with social media, followed by its print circulation and the preexisting print audience. In other words, Twitter popularity may become self-reinforcing. As magazines make an effort to become more active on Twitter, they gain more followers, who then encourage more activity on Twitter. Overall, however, these results likely boil down to the readily apparent fact that larger magazines have more resources to engage in social media activity than do smaller magazines, such as the ability to hire dedicated social media staff. As such, they are more likely to build large Twitter followings and tweet often.

Finally, as one would expect, there was a moderate and significant correlation between the average household income of a magazine's print readership and its number of Twitter followers. Though most city magazines attract affluent audiences, one might expect that the wealthiest readers will have greater access to the technology needed for Twitter, as well as leisure time for its use. In general, however, given the rapidly changing nature of social media and audience adoption of this still new technology, these initial analyses can only be exploratory.

\section{Discussion}

This study sought to explore how Twitter might be affecting city magazines' role in informing local audiences. The study found that so far on Twitter, city magazines are still largely addressing a variety of topics and local concerns similar to their established formulas of content, specifically their focus on entertainment and food. Remarkably, these findings are consistent with those of studies on print city magazines conducted three decades ago. In addition, this study found that these city magazines are not really capitalizing on the unique capabilities of Twitter as a medium. They are not 
sharing multimedia, as is so common in social media, nor are they promoting their print or digital products and activities extensively through tweets. The city magazines appear to be using Twitter primarily as a broadcasting tool to generate website traffic through links, as opposed to a conversational tool to engage audiences deeply and generate discussion.

However, it is notable that these magazines are using Twitter frequently to share information on timely topics that are of value to their audiences at the moment of sharing, as represented by the occurrence of words such as "today" and "this weekend" in their tweets. Moreover, they are using their online content as a primary destination for their Twitter audiences. The capability of online content and social media to engage audiences immediately-instead of on a monthly or bimonthly print publication schedule-is something new for city magazines and is a development on which much can be built in the near future.

Some city magazines have begun to integrate a wider variety of topics into their print editions (as a complementary portion of this research project has shown), thereby better supplementing fading newspaper coverage in many cities. These pioneering publications might also consider ways in which their social media outreach can enhance their coverage of these and more typical topics in order to better inform and activate local (and broader) audiences regarding the issues they cover. All city magazines can also use social media, including Twitter, to create more audience loyalty by becoming even more integral to the satisfaction of audiences' daily local information needs. The results of this study showed that city magazines have generally not conversed much with other Twitter users, even in the form of merely mentioning their names. However, other Twitter users appear to have a positive sense of these magazines, as the word cloud analysis showed. Therefore, city magazines might both broaden and intensify their social media efforts to build on that positive reputation and better serve their local audiences.

In a publishing environment in which building paid print circulation still brings the greatest rewards in terms of advertising revenue, it may be difficult for city magazines to imagine or quantify the return on investment in social media outreach. After all, more Twitter followers may not immediately lead to more print subscribers. However, as audiences continue to shift their time and subscription dollars toward digital and mobile media, advertisers will follow. City magazines can prepare for this shift by ensuring that they have built active, engaged, and enthusiastic social media audiences who will be more likely to consume their digital products as well. This shift will also likely gain momentum as younger audiences - many of who have never subscribed to print magazinesmature and begin to seek more substantial sources of local information to enable them to better participate in their communities. Investing in social media, which can reach these youthful audiences today and prepare them for continuing engagement with magazines in the future, will likely have long-term benefits for city magazines.

This study is admittedly preliminary and incomplete without a broader look at the production and content of city magazines' print editions, which is forthcoming in a broader research project now under way. This analysis is also limited in that it looked at just a few aspects of the magazines' Twitter efforts, offered a detailed analysis of only a small sample of tweets, did not include other social media (particularly Facebook), and could not compare the city magazines' tweets as they happened 
concurrently with their print and Web content. All of these are areas for exploration in future research.

Another element that could not be analyzed in this study was the links contained in the city magazines' tweets. Because of the ways in which links are shortened on Twitter and the limited capabilities of the data-collection service used in this study, it was not possible to analyze which sites the magazines chose to link to in their tweets (beyond noting the frequency of their mentions of their own affiliates' Twitter usernames). An analysis of these links might have revealed further insight into the types of content that city magazines select and promote to their audiences. Given researchers' interest in aggregation and curation in today's journalism (e.g., Stanoevska-Slabeva, Sacco, \& Giardina, 2012), such analysis would be useful for comparing and contrasting city magazines' activities in this regard with those of other journalistic media. Future analyses of city magazines' social media might also examine in greater detail the specific interactions between audience members and magazine staffers through social media, such as commentary on a particular topic/story or feedback related to crowd-sourcing efforts, to assess how city magazines use the medium to address particular topics and relate to their audiences.

\section{Conclusion}

Magazine scholars are well aware of the ways in which magazines help to shape and create communities around the subjects they cover, and city magazines_often dismissed as lifestyle fluffmay be increasingly important in constituting and informing local audiences, especially when armed with new digital tools. Though this study has not revealed any great shift in city magazines' presentday role that might be visible through their use of Twitter, it can serve as a benchmark for evaluating future advances. It also hints at an exciting future for a medium that has seen very little change in the last few decades. City magazines can now extend their work into the local information gaps created by the decline of traditional news providers and can also begin to distribute that work to their audiences instantaneously in a variety of digital forms. With those changes, city magazines' content, purpose, and role may soon begin to shift dramatically. Freed from the constrictions of the print format and a monthly publication schedule, a realm of new opportunities awaits if city magazines can seize this potential. This is certainly a medium worth following.

\section{References}

DiscoverText. (2012). DiscoverText: A text analytic toolkit for ediscovery and research. Retrieved from http://discovertext.com/

Federal Communications Commission. (2011). The information needs of communities: The changing media landscape in a broadband age. Retrieved from http://www.fcc.gov/info-needscommunities

Fletcher, A. D., \& VandenBergh, B. G. (1982). Numbers grow, problems remain for city magazines. Journalism Quarterly, 59(2), 313-317. doi: 10.1177/107769908205900222 
Greenberg, M. (2000). Branding cities: A social history of the urban lifestyle magazine. Urban Affairs Review, 36(2), 228-263. doi: 10.1177/10780870022184840

Hayes, J. P. (1981). City/regional magazines: A census. Journalism Quarterly, 58(2), 294-296. doi: $10.1177 / 107769908105800219$

Hermida, A. (2012). Social journalism: Exploring how social media is shaping journalism. In E. Siapera \& A. Veglis, The handbook of global online journalism (pp. 309-328). Hoboken, NJ: John Wiley \& Sons.

Holcomb, J., Gross, K., and Mitchell, A. (2011). How mainstream media outlets use Twitter (Project for Excellence in Journalism survey). Retrieved from http://www.journalism.org/analysis_report/how_mainstream_media_outlets_use_twitter

Hynds, E. C. (1979). City magazines, newspapers serve in different ways. Journalism Quarterly, 56(3), 619-622. doi: 10.1177/107769907905600324

Phelps, A. (2012). Why the Denver Post is putting more local news on A1. Nieman Journalism Lab. Retrieved from http:/www.niemanlab.org/2012/04/why-the-denver-post-is-putting-morelocal-news-on-a1/

Project for Excellence in Journalism. (2012a). 72\% of Americans follow local news closely. Retrieved from http://www.journalism.org/commentary_backgrounder/72_ americans_follow_local_news_closely

Project for Excellence in Journalism. (2012b). State of the news media 2012: An annual report on American journalism. Retrieved from http://stateofthemedia.org/

Stanoevska-Slabeva, K., Sacco, V., \& Giardina, M. (2012). Content curation: A new form of gatewatching for social media? Paper presented at the International Symposium on Online Journalism, Austin, TX.

Takhteyev, Y., Gruzd, A., \& Wellman, B. (2011). Geography of Twitter networks. Social Networks, 34, 73-81. doi: 10.1016/j.socnet.2011.05.006

Twitter. (2012). What are hashtags (“\#” symbols)? Retrieved from https://support.twitter.com/articles/49309-what-are-hashtags-symbols

\section{Acknowledgments}

I would like to thank Lisa Weidman, assistant professor of mass communication at Linfield College, for her thoughtful feedback on this study. I am also grateful for the assistance of Jaimie McDonald, my undergraduate research assistant during summer 2012. 